\title{
Quartic Trigonometric Tension B-spline Finite Element Method for Solving Gardner Equation
}

\author{
Ozlem Ersoy Hepson ${ }^{1}$ \\ ${ }^{1}$ Eskisehir Osmangazi Universitesi Fen Edebiyat Fakultesi
}

April 27, 2020

\begin{abstract}
Method of collocation is established for getting solution of the Gardner equation (GE). The GE is fully integrated by way of the Crank-Nicolson method for time variable and collocation method for spatial variable. Trial function of the collocation method is set up using combination of quartic trigonometric tension (QTT) B-splines. Convergence analysis of suggested method is investigated. Performance of the quartic trigonometric tension B-splines is searched by studying three test problems; propagation of bell shape solitary wave, interaction of two positive bell shape solitary wave and wave generation.
\end{abstract}

Hosted file wileyNJD-AMA.pdf available at https://authorea.com/users/306419/articles/445581-quartic-trigonometrictension-b-spline-finite-element-method-for-solving-gardner-equation 
figures/Fig1/Fig1-eps-converted-to.pdf 
figures/Fig2a/Fig2a-eps-converted-to.pdf 
figures/Fig2b/Fig2b-eps-converted-to.pdf 
figures/Fig3a/Fig3a-eps-converted-to.pdf 
figures/Fig3b/Fig3b-eps-converted-to.pdf 
figures/Fig3c/Fig3c-eps-converted-to.pdf 
figures/Fig3d/Fig3d-eps-converted-to.pdf 
figures/Fig3e/Fig3e-eps-converted-to.pdf 
figures/Fig3f/Fig3f-eps-converted-to.pdf 
figures/Fig4a/Fig4a-eps-converted-to.pdf 
figures/Fig4b/Fig4b-eps-converted-to.pdf 
figures/Fig4c/Fig4c-eps-converted-to.pdf 
figures/Fig4d/Fig4d-eps-converted-to.pdf 